\title{
Formation of an fcc phase through a bcc metastable state in crystallization of charged colloidal particles
}

\author{
Shenghua Xu, ${ }^{1,2}$ Hongwei Zhou, ${ }^{1,2}$ Zhiwei Sun, ${ }^{1,2, *}$ and Jingchang $\mathrm{Xie}^{1,2}$ \\ ${ }^{1}$ Key Laboratory of Microgravity, Institute of Mechanics, Chinese Academy of Sciences, 100190 Beijing, People's Republic of China \\ ${ }^{2}$ National Microgravity Laboratory, Institute of Mechanics, Chinese Academy of Sciences, 100190 Beijing, People's Republic of China
}

(Received 8 March 2010; revised manuscript received 22 April 2010; published 20 July 2010)

\begin{abstract}
By in situ monitoring structural changes with the reflection spectrometer during the colloidal crystallization, we present direct experimental evidence of liquid-bcc-fcc phase transition in crystallization of charged colloidal particles, as a manifestation of the Ostwald's step rule. In addition, the lifetime of the bcc metastable structure in this system decreases significantly with increasing particle volume fraction, offering a possible explanation for "exceptions" to the step rule.
\end{abstract}

DOI: 10.1103/PhysRevE.82.010401

PACS number(s): 64.60.My, 64.70.dg, 64.70.pv, 82.70.Dd

In recent years, colloidal crystals have received great attention as model systems that mimic atoms and molecules. The colloidal crystallization has been used to study basic aspects of phase transitions at much larger time and length scales [1-4]. In a common strategy, the phase behavior and crystallization kinetics of colloidal particles are used to compare with their atomic or molecular counterparts and to assess the validity of classical crystal growth theories.

Understanding the nature of the structure's evolution during the nucleation and growth of crystals is still a fundamental and challenging issue in condensed-matter science. Various efforts have been devoted to deepening this understanding. As an important empirical rule for the structure's evolution, the Ostwald's step rule [5] was first proposed in 1897, based on previously available observations. However, a strict theoretical proof is still lacking although a great deal of effort has been devoted in finding a theoretical basis for the rule. According to this rule, the solid phase first formed in the crystallization process would be the least stable polymorph, instead of the stable one.

Many years later, on the basis of the mean-field treatment, Alexander and McTague [6] further indicated that a bcc structure should be formed first regardless of whether a more thermodynamically stable one exists, as long as the firstorder nature of the transition from the liquid is not too distinct. Since then, many attempts have been made to find evidence supporting the existence of the metastable bcc phase. Some computer simulations have demonstrated the bcc-fcc structural transition [7-10]. Experimentally, on the other hand, the metastable bcc phase has been observed in rapidly quenched metals $[11,12]$ and block copolymer solution $[13,14]$. However, the behavior of the bcc-fcc structural transition in the colloidal crystallization has not been explicitly exhibited $[1,14,15]$, although it was observed that the bcc pattern always occurred first before the fcc pattern appeared for the charged latex particles at moderate number density $[16,17]$.

In this Rapid Communication, we present direct experimental evidence of liquid-bcc-fcc phase transition in crystallization of charged colloidal particles by using the reflection

\footnotetext{
*Corresponding author. sunzw@imech.ac.cn
}

spectrum (RS) to identify the type of colloidal crystal structures. The bcc-fcc transition was confirmed by bcc-decay rate being equal to fcc-growth rate. We further show that the lifetime of the bcc metastable structure for this system strongly depends on particle volume fraction.

When the phase-change process is too fast to observe, one may not be able to see what really happens during the structural evolution. It would be interesting here to recall Ostwald's statement: "it is easy to formulate such a hypothesis, yet not always possible to prove it with existing techniques. However, in many of these cases it will be possible to find the appropriate means to slow down the reaction (i.e. the phase transformation) to enable the observation of the intermediate phase" $[5,18]$.

For charged colloidal dispersions, their crystallization process is tunable by properly adjusting the particle volume fraction $\Phi$ and the strength of the repulsion between particles. We therefore chose them to be the object of study to make the pace of phase change more suitable based on the measurement capability. In this study, negatively charged polystyrene (PS) spheres are adopted.

The reflection spectrum has been applied in measurements of the lattice distance [19-21], as well as the nucleation and growth rates [22]. We have found that if all reflection peaks distributed in the wavelength $(\lambda)$ space are transferred into the wave-vector $(q)$ space, where $q=\frac{4 \pi \mathrm{n}}{\lambda} \sin (\theta / 2)$, they also can be used to identify the type of crystal structures. This procedure is similar to the static light scattering (SLS) method, in which the scattering intensity at different wave vectors $q$ is measured to determine the crystal structures. For conventional SLS, the wavelength $\lambda$ (defined by the laser light source used in the measurement) is kept constant, while the scattering angle $\theta$ is changed by mechanically rotating the goniometer during measurement. In contrast, for $\mathrm{RS}, \theta$ is a constant $\left(\theta=180^{\circ}\right.$ in this study) during the measurement but $\lambda$ is changing by spectrum scanning. Apparently, the signals of the reflection spectrum are mainly contributed by the colloids next to boundary of the crystallization cell. Therefore, the crystal structural changes detected in the measurements are supposedly corresponding to heterogeneous crystallization near the cell wall.

In the experiment for this study, the PS microspheres were synthesized by an emulsion polymerization method [23]. 


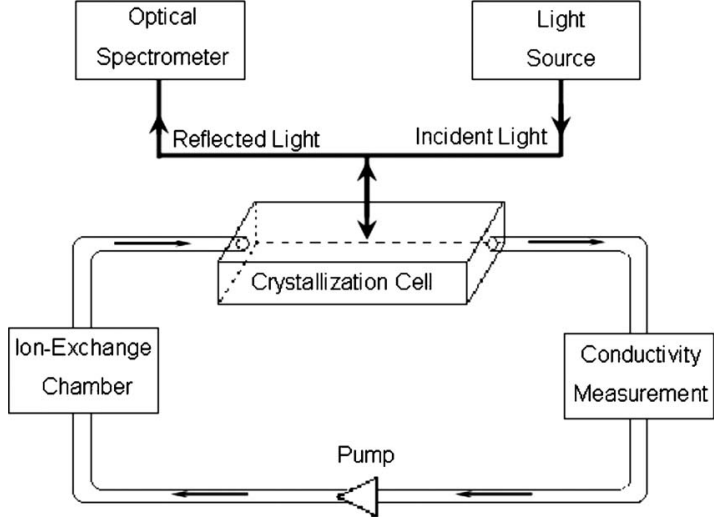

FIG. 1. Schematic of the experimental setup, which consists of a crystallization cell, a reflection spectrometer, a circulating pump, an ion-exchange chamber and a conductivity measurement unit. The data of light intensities reflected from the sample in the crystallization cell for different wavelengths are collected by the reflection spectrometer. The pump is used to shear melt the crystals and the iox-exchange chamber is used to keep a low ion concentration in the sample.

Thereafter, purification of the spheres was performed by filtration and followed by repeated washing with fresh distilled water in the centrifugation process. The mean diameter and polydispersity of the microspheres are $101 \mathrm{~nm}$ and $4 \%$, respectively. The titrated surface charge density of the microspheres are $9.9 \mu \mathrm{C} \mathrm{cm}^{-2}$ and the effective value calculated according to the empirical relationship [24] is $2.6 \mu \mathrm{C} \mathrm{cm}^{-2}$. We did not add any additional salt in the suspension and the foreign ions contained in the sample were removed by ionexchange resin.

As schematically shown in Fig. 1, the experimental setup included a suspension circulation system, which consists mainly of a crystallization cell, a reflection spectrometer, a circulating pump, an ion-exchange chamber and a conductivity measurement unit. This circulation system is similar to those described in Refs. [20,25]. Here a fiber optic spectrometer (Avaspec-2048, Avantes, Netherlands) with a tungsten halogen light source (Avalight-HAL, Avantes, Netherlands) and a bifurcated fiber optic cable was used to scan the light intensity reflected from the crystallization cell over a wavelength range. Data, including the distribution of reflection intensity vs wavelength, were recorded at the sampling rate of 4 spectra per second. Therefore, the structure change process can be monitored in situ with a time resolution as fast as $0.25 \mathrm{~s}$. The range of $q$ that can be provided by the spectrometer is about $17-50 \mu \mathrm{m}^{-1}$. The shear flow driven by the pump can easily destroy (melt) crystal structures preformed in the crystallization cell. Circulating the suspension through ion-exchange resin can help the system stay in a state with low ion concentration. The crystallization process begins just after cessation of shear flow (we approximately assume that at this moment the crystallization time $=0$ ) and the data on time-dependent reflection intensity from the sample during the crystallization can be recorded. This procedure can be repeated.

Figure 2 shows the reflection spectra measured at crystallization time $=0.8 \mathrm{~min}, 22 \mathrm{~min}$ and $65 \mathrm{~min}$, respectively, for

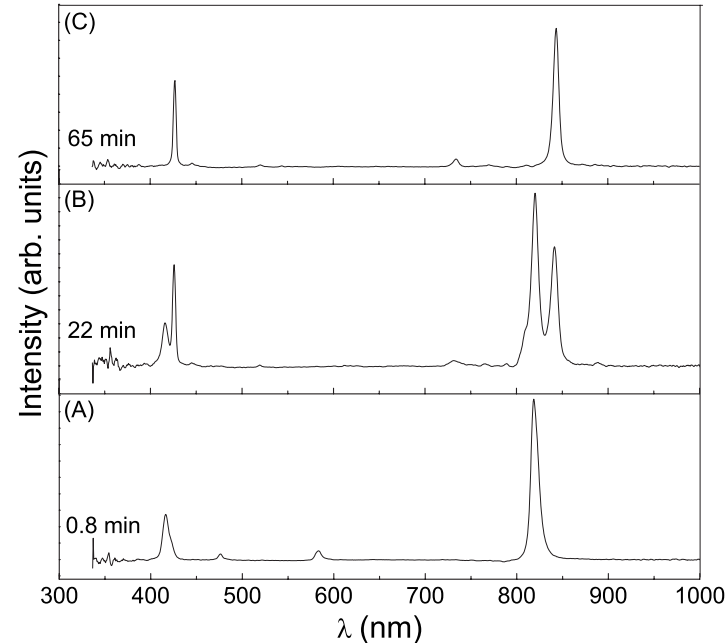

FIG. 2. Reflected (or Bragg scattered) intensity vs $\lambda$ for $\Phi=0.75 \%$ at different crystallization time: (a) $0.8 \mathrm{~min}$; (b) $22 \mathrm{~min}$; (c) $65 \mathrm{~min}$.

the particle volume fraction $=0.75 \%$. When the plot in Fig. 2 is converted to that in the wave-vector space, we have the plot in Fig. 3 where the $x$ axis becomes wave vector $q$ instead of wavelength $\lambda$. For the spectrum at $0.8 \mathrm{~min}$, the position of the reflected peaks in wave-vector space are 20.4, 28.7, 35.2, and $40.2 \mu \mathrm{m}^{-1}$, respectively, and the ratio of them is $\sqrt{2}: \sqrt{4}: \sqrt{6}: \sqrt{8}$. Therefore, the crystal structure that appeared is bcc, and the corresponding peaks were assigned to (110), (200), (211), and (220) crystal planes. Similarly, for the spectrum at $65 \mathrm{~min}$ in Fig. 3 the crystal structure becomes fcc only, and the relevant peaks are from (111), (200), (220), and (222) planes, respectively. However, at the crys-

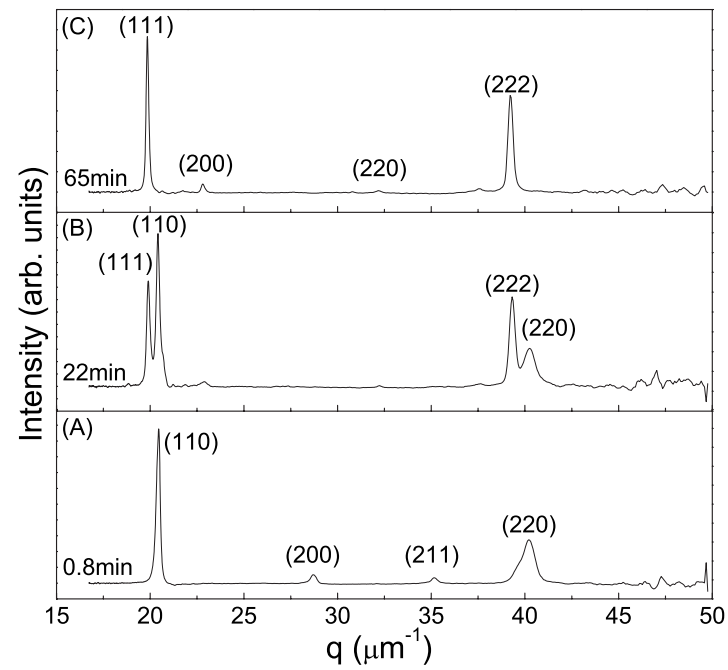

FIG. 3. Bragg scattered intensity vs $q$ for $\Phi=0.75 \%$ at different crystallization time: (a) $0.8 \mathrm{~min}$. The crystal structure is bcc and the corresponding peaks are from (110), (200), (211), and (220) crystal planes. (b) $22 \mathrm{~min}$. The coexistence of metastable bcc and fcc structures and the (111) plane of fcc structure and (110) plane of bcc structure are somewhat overlapped. (c) $65 \mathrm{~min}$. The crystal structure is fcc and corresponding peaks are from (111), (200), (220) and (222) planes. 


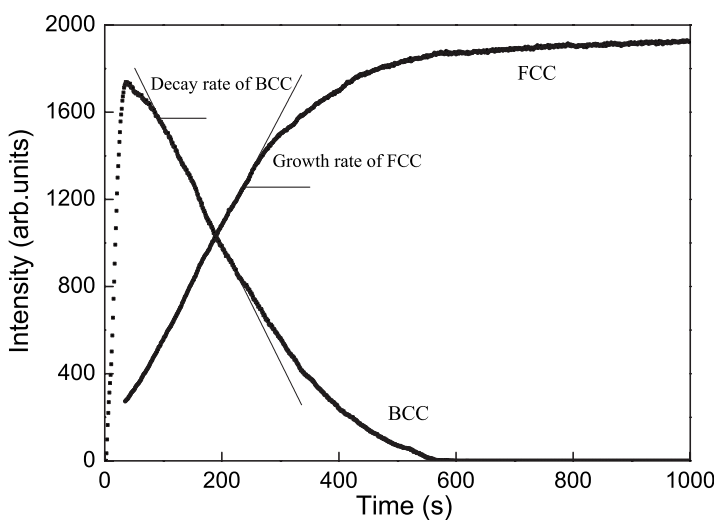

FIG. 4. The change of peak intensities from (111) plane of fcc structure and (110) plane of bcc structure with time. The volume fraction is $0.92 \%$. The growth rate of fcc structure and the decay rate of bcc structure during bcc-fcc transition can be evaluated from the slop of the curves, as is shown in the figure.

tallization time in between, for the spectrum at $22 \mathrm{~min}$, the bcc and fcc structures coexist so that peaks from (111) plane of fcc structure and (110) plane of bcc structure are somewhat overlapped and form a double peak structure as depicted in Fig. 3. From these spectrum shots at 0.8, 22, and 65 min, we can see that the metastable bcc structure nucleates and grows first, then gradually transforms to the stable fcc phase until the whole transformation process finishes with only fcc structure left in the sample.

Figure 4 shows the evolution of peak intensities for bcc and fcc during the crystallization at volume fractions $0.92 \%$, where two curves are associated with the fcc (111) plane and the bcc (110) plane, respectively. As shown, the bcc structure emerges first and rapidly reaches its maximum value, then drops down. On the other hand, in Fig. 4 the fcc structure appears only after a period of the crystallization time. The reason is twofold: first, the fcc structure can only transform from the bcc structure instead of being formed alone, so fcc needs more time to form and grow; second, at the early stage of the bcc-fcc transition, peaks of the fcc (111) plane are too weak to distinguish from the coverage of the adjacent peaks of bcc structure. That is, the fcc structure is not detectable at the early stage of the crystallization. This is due to limitations with our detecting method, although it actually should start earlier than shown in Fig. 4.

From Fig. 4 we can also evaluate the growth rate of fcc structure and the decay rate of bcc structure from the slope of the curves during structure transition, following the method in Ref. [26]. The growth rate of fcc structure and the decay rate of bcc structure are $5.1 \mathrm{~s}^{-1}$ and $-5.4 \mathrm{~s}^{-1}$, respectively, evaluated from Fig. 4. Apparently, these two rates are very close in magnitude, so that the estimated conversion rate of bcc-fcc is approximately $5.3 \mathrm{~s}^{-1}$. It is clear that the fcc structure grows at the expense of bcc structure until the bcc structure totally vanishes and the size of fcc structure reaches a constant value.

From the Bragg peaks, the crystallinity $X(t)$ (the fraction of the sample which is crystalline), the average linear dimension of the crystallites, and the lattice constants, etc. can be evaluated [27]. Since our major concern is the transition pro-

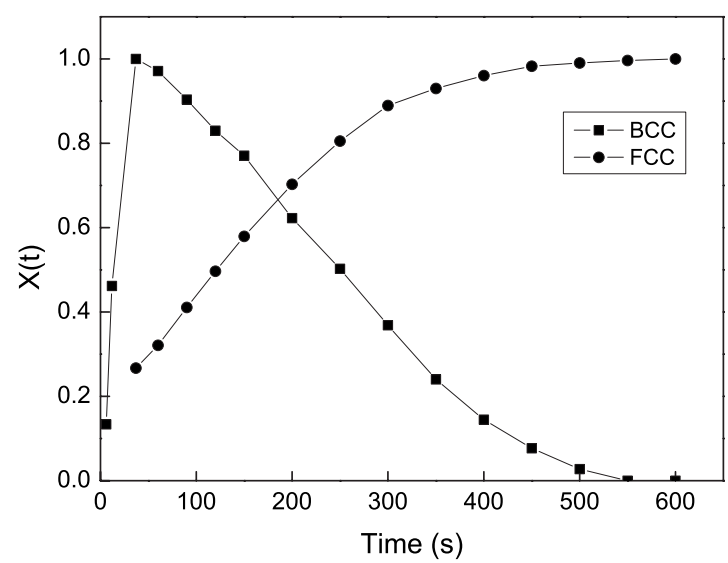

FIG. 5. The crystallinity $X(t)$ of fcc and bec structure during the bec-fcc transition. The volume fraction is $0.92 \%$.

cess, the crystallinity of both bcc and fcc structures during the bcc-fcc transition is presented in Fig. 5. The value of $X(t)$ is calculated from the peak area $A(t)$ by

$$
X(t)=c A(t),
$$

where $c$ is normalization factor. For bcc and fcc structures, the peaks from fcc (111) plane and the bcc (110) plane are used in calculating their relevant values of $A(t)$. And the normalization factor for bcc is based on the maximum value of peak area of bcc (110) plane during crystallization, while the normalization factor for fcc is based on the equilibrium phase. From Fig. 5 we can see that the crystallinity of bcc first increases very fast and then drops down with the increase of crystallinity of fcc structure. The shapes of curves shown in Figs. 5 and 4 are quite similar, confirming again that the fcc structure is transited from bcc structure, and thus supporting the Ostwald's step rule [5] and the theoretical prediction of Alexander and McTague [6].

To study the dependence of the lifetime of the bcc metastable state on the volume fraction, we further performed more experiments on the phase transition process at different volume fractions. Some important data are listed in Table I: the equilibrium structure, and (a) the time for bcc structure to reach its maximum size; (b) the time for the bcc structure to vanish; and (c) the time for fcc structure to reach its stable state, for four different volume fractions $0.39 \%, 0.57 \%$,

TABLE I. Equilibrium structure and related time during structure evolution for different volume fractions.

\begin{tabular}{|c|c|c|c|c|}
\hline $\begin{array}{l}\text { Volume } \\
\text { fraction }\end{array}$ & $\begin{array}{l}\text { Equilibrium } \\
\text { structure }\end{array}$ & $\begin{array}{c}\text { (a) } \\
\text { The time } \\
\text { for bcc } \\
\text { to reach } \\
\text { maximum(s) }\end{array}$ & $\begin{array}{l}\text { (b) } \\
\text { The time } \\
\text { for bcc } \\
\text { to vanish }\end{array}$ & $\begin{array}{c}(\mathrm{c}) \\
\text { The time } \\
\text { for fcc } \\
\text { to reach } \\
\text { maximum }\end{array}$ \\
\hline $0.39 \%$ & bcc & 200 & N/A & N/A \\
\hline $0.57 \%$ & fcc & 50 & $\sim 9 \mathrm{~h}$ & $\sim 9 \mathrm{~h}$ \\
\hline $0.75 \%$ & fcc & 36 & $50 \mathrm{~min}$ & $50 \mathrm{~min}$ \\
\hline $0.92 \%$ & fcc & 15 & $9 \min$ & $9 \min$ \\
\hline
\end{tabular}


$0.75 \%$, and $0.92 \%$, respectively. For volume fractions $0.39 \%$, the stable structure is bcc alone so items (b) and (c) are not available. For other three volume fractions, the stable structure is fcc, and all the liquid-fcc phase transitions pass through a metastable bcc state. Also, as discussed above, the times for (b) and (c) are actually the same for a given volume fraction.

The change tendency of the structure evolution vs the volume fraction is clear: the crystallization time and the lifetime of the metastable bcc state decrease with the increase of the volume fraction. The lifetime of the metastable bcc state can range from several minutes to about $10 \mathrm{~h}$ depending on the volume fraction.

Both theoretical results [28] and experimentally obtained phase diagram [29] from previous studies have explained the phase behavior for a charged colloidal system in an equilibrium state. That is, at lower volume fraction bcc is stable structure and at higher volume fraction the stable structure becomes fcc when other variables such as the ion concentration or interaction forces are kept constant, which is coincident with Table I. According to our observations described above, we can presume that the difference in free energy between the stable fcc state and the bcc state, whose free energy is closest to that of a fluid phase (which should be first nucleated according to the Ostwald's step rule), is larger for a larger volume fraction. This free energy difference would drive the liquid-bcc-fcc transition faster for larger volume fractions. Particularly, from this tendency it seems reasonable to speculate that a metastable bcc state might also exist for crystallization in hard-sphere suspensions and this metastable state has not been seen only because its lifetime is too short to detect due to a much higher volume fraction (between 0.545 and 0.58 ) $[15,30,31]$ necessary for the crystallization.
In summary, analyzing the reflection spectrum in the wave-vector space makes it possible to identify the type of crystal structures during the colloidal crystallization. Taking advantage of this new methodology, our experiments have shown that the liquid-bcc-fcc phase transition does exist in heterogeneous crystallization process near the cell wall for charged colloidal particles. This finding is not only a manifestation of the Ostwald's step rule but also direct evidence for what predicted by Alexander and McTague [6]. Furthermore, our data demonstrate that the lifetime of the bcc metastable structure for this system decreases significantly with increasing particle volume fraction. It seems as if there was a volume-fraction window for observers to see the bcc-fcc transition. At one end, the associated volume fraction is too high and the bcc-fcc transition happens too quickly to follow, with the result that only fcc can be seen. At the other end, the volume fraction is low and the bcc lifetime is either too long or bcc actually becomes a stable structure. If this conjecture is true, it may explain why the bcc-fcc transition cannot be seen in many cases although it might nonetheless exist. Particularly, our finding actually also coincides with Ostwald's argument against occasional exceptions to the rule: "...there will be cases where for a given phase transformation, a metastable phase exists, but is not observed. In those cases one may always assume that this intermediate phase does form, but transforms immediately (into the stable phase)" [18].

We thank Dr. Daan Frenkel for promptly offering his translation [18]. This work is supported by Grants No. 10672173, No. 10972217, and No. 10932012 from the National Natural Science Foundation of China and the Knowledge Innovation Program of the Chinese Academy of Sciences (including Grant No. KJCX2-YW-L08).
[1] V. J. Anderson and H. N. W. Lekkerkerker, Nature (London) 416, 811 (2002).

[2] M. E. Leunissen et al., Nature (London) 437, 235 (2005).

[3] A. P. Hynninen and M. Dijkstra, Phys. Rev. Lett. 94, 138303 (2005).

[4] A. Yethiraj and A. van Blaaderen, Nature (London) 421, 513 (2003).

[5] W. Ostwald, Z. Phys. Chem. 22, 289 (1897).

[6] S. Alexander and J. McTague, Phys. Rev. Lett. 41, 702 (1978).

[7] P. R. ten Wolde, M. J. Ruiz-Montero, and D. Frenkel, Phys. Rev. Lett. 75, 2714 (1995).

[8] Y. C. Shen and D. W. Oxtoby, Phys. Rev. Lett. 77, 3585 (1996).

[9] P. G. Bolhuis et al., Nature (London) 388, 235 (1997).

[10] C. Desgranges and J. Delhommelle, Phys. Rev. Lett. 98, 235502 (2007).

[11] C. Notthoff, B. Feuerbacher, H. Franz, D. M. Herlach, and D. Holland-Moritz, Phys. Rev. Lett. 86, 1038 (2001).

[12] G. Ghosh, Mater. Sci. Eng., A 189, 277 (1994).

[13] Y. Liu et al., Phys. Rev. E 73, 061803 (2006).

[14] J. Bang and T. P. Lodge, Phys. Rev. Lett. 93, 245701 (2004).

[15] U. Gasser, J. Phys.: Condens. Matter 21, 203101 (2009).

[16] H. J. Schope, T. Decker, and T. Palberg, J. Chem. Phys. 109,

\section{8 (1998).}

[17] T. Palberg, J. Phys.: Condens. Matter 11, R323 (1999).

[18] The translation from [5] is provided by Dr. Daan Frenkel in a private communication.

[19] T. Okubo, J. Chem. Soc., Faraday Trans. 82, 3163 (1986).

[20] T. Okubo and H. Ishiki, J. Colloid Interface Sci. 228, 151 (2000).

[21] L. Liu et al., J. Colloid Interface Sci. 326, 261 (2008)

[22] T. Okubo et al., Colloid Polym. Sci. 280, 290 (2002).

[23] G. T. D. Shouldice, G. A. Vandezande, and A. Rudin, Eur. Polym. J. 30, 179 (1994).

[24] H. Yoshida et al., Langmuir 15, 2684 (1999).

[25] T. Palberg et al., J. Phys. Chem. 96, 8180 (1992).

[26] J. K. G. Dhont, C. Smits, and H. N. W. Lekkerkerker, J. Colloid Interface Sci. 152, 386 (1992).

[27] P. Wette et al., J. Phys.: Condens. Matter 21, 464115 (2009).

[28] M. O. Robbins, K. Kremer, and G. S. Grest, J. Chem. Phys. 88, 3286 (1988).

[29] E. B. Sirota et al., Phys. Rev. Lett. 62, 1524 (1989).

[30] P. N. Pusey and W. van Megen, Phys. Rev. Lett. 59, 2083 (1987)

[31] W. G. Hoover and F. H. Ree, J. Chem. Phys. 49, 3609 (1968). 